OPEN ACCESS

Edited by:

Paolo Vineis,

Imperial College London,

United Kingdom

Reviewed by:

Jeff Bolles,

University of North Carolina at Pembroke, United States

Piercarlo Sarzi Puttini,

University of Milan, Italy

*Correspondence:

Claire Colas

claire.colas@univ-st-etienne.fr

Specialty section:

This article was submitted to

Public Health Education and

Promotion,

a section of the journa

Frontiers in Public Health

Received: 23 April 2020

Accepted: 29 March 2021

Published: 25 May 2021

Citation:

Colas C, Goutte J, Creac'h C, Fontana L, Vericel M-P, Manzanares J,

Peuriere M, Akrour M, Martin C,

Presles E, Barth N, Guyot J, Garros M,

Trombert B, Massoubre $C$, Roche F,

Féasson L, Marotte H, Cathebras $P$ and Hupin D (2021) Efficiency of an

Optimized Care Organization in Fibromyalgia Patients: The From Intent to Move (FIMOUV) Study Protocol of a

Randomized Controlled Trial.

Front. Public Health 9:554291.

doi: 10.3389/fpubh.2021.554291

\section{Efficiency of an Optimized Care Organization in Fibromyalgia Patients: The From Intent to Move (FIMOUV) Study Protocol of a Randomized Controlled Trial}

Claire Colas ${ }^{1,2 *}$, Julie Goutte ${ }^{3}$, Christelle Creac' $h^{4,5}$, Luc Fontana $^{6,7}$, Marie-Pierre Vericel $^{2}$, Jessica Manzanares ${ }^{4}$, Marie Peuriere ${ }^{8}$, Madjid Akrour ${ }^{8}$, Charly Martin ${ }^{8}$, Emilie Presles ${ }^{1,8,9}$, Nathalie Barth ${ }^{1,10,11}$, Jessica Guyot ${ }^{1,10}$, Maël Garros ${ }^{12}$, Béatrice Trombert ${ }^{1,13}$, Catherine Massoubre ${ }^{14}$, Frédéric Roche ${ }^{1,2}$, Léonard Féasson ${ }^{2,15}$, Hubert Marotte ${ }^{1,16}$, Pascal Cathebras ${ }^{3}$ and David Hupin ${ }^{1,2,17}$ on behalf of the FIMOUV investigators

1 University Lyon, UJM-Saint-Etienne Sainbiose Laboratory, INSERM U1059, Saint-Étienne, France, ${ }^{2}$ Department of Clinical and Exercise Physiology, University Hospital Center, Saint-Étienne, France, ${ }^{3}$ Department of Internal Medicine, University Hospital Center, Saint-Étienne, France, ${ }^{4}$ Pain Center, University Hospital Center, Saint-Étienne, France, ${ }^{5}$ University Claude Bernard, Central Integration of Pain (NeuroPain) Lab-Lyon Neuroscience Research Center, INSERM U1028, CNRS, Bron, France, ${ }^{6}$ Department of Occupational and Environmental Medicine, University Hospital Center, Saint-Étienne, France, ${ }^{7}$ University Lyon, University Lyon 1, University St Etienne, University Gustave Eiffel, IFSTTAR, UMRESTTE, UMR_T9405, Saint-Étienne, France, ${ }^{8}$ Clinical Research, Innovation and Pharmacology Unit, University Hospital Center, Saint-Étienne, France, ${ }^{9}$ Department of Clinical Investigation Center, CIC 1408-INSERM, University Hospital Center, Saint-Étienne, France, ${ }^{10}$ University Lyon, UJM-Saint-Etienne Chaire Santé des Ainés, Saint-Étienne, France, ${ }^{11}$ Gerontopole Auvergne-Rhone-Alpes, Saint-Étienne, France, ${ }^{12}$ Sport Health House, CDOS 42, Saint-Étienne, France, ${ }^{13}$ Department of Public Health, University Hospital Center, Saint-Étienne, France, ${ }^{14}$ Department of Psychiatry, University Hospital Center, Saint-Étienne, France, ${ }^{15}$ University Lyon, UJM-Saint-Etienne Interuniversity Laboratory of Human Movement Biology, EA 7424, Saint-Étienne, France, ${ }^{16}$ Department of Rheumatology, University Hospital Center, Saint-Étienne, France, ${ }^{17}$ Department of Medicine, K2, Solna Karolinska Institutet, Stockholm, Sweden

Introduction: Fibromyalgia (FM) is characterized by multiple symptoms including pain, fatigue, and sleep disorders, altering patient's quality of life. In the absence of effective pharmacological therapy, the last European guidelines recommend a multidisciplinary management based on exercise and education. Thus, our main objective was to measure the effectiveness of a healthcare organization offering a specific program of adapted physical activity combined with a therapeutic education program for FM patients.

Methods and Analysis: The From Intent To Move (FIMOUV) study will recruit 330 FM patients randomized into two groups: test and control. The test group will benefit from a 1-month mixed exercise training program supervised the hospital, followed by 2 months in a community-based relay in a health-sport structure. In addition, each of the two groups will benefit from therapeutic patient education sessions. The main endpoint is the measurement of the level of physical activity by accelerometry at 1 year. The secondary endpoints concern adherence to the practice of physical activity, impact on lifestyle, state of health, and physical capacity, as well as an estimate of the budgetary impact of this management strategy. 
Discussion: This interventional research will allow us to assess the evolution of behaviors in physical activity after an FM syndrome management based solely on patient education or based on a supervised and adapted practice of physical activity associated with this same therapeutic education program. It seems to be the first study evaluating the impact of its intervention on objective data for measuring physical activity and sedentary behavior via accelerometry among FM patients.

Trial registration: ClinicalTrials.gov NCT04107948.

Keywords: fibromyalgia, exercise, patient education, behavior in physical activity, accelerometry, care organization

\section{INTRODUCTION}

Fibromyalgia (FM) affects $2-5 \%$ of the adult population, with a feminine predominance (1). This syndrome is characterized by multiple symptoms including pain, fatigue, and sleep disorders, altering patient's quality of life (2). Physiopathology is still poorly understood, but a dysfunction of the central nervous system is evoked, involving various mechanisms of pain pathways (3). Diagnosis of FM is based on the American College of Rheumatology criteria $(4,5)$ and associates a quantitative pain rating scale (Widespread Pain Index) and a rating scale of the severity of symptoms associated with pain (Symptom Severity Scale). According to these last recommendations (4), FM diagnosis is no more an elimination diagnosis but rests on the scores of these two scales, the chronicity of pain $(>3$ months), the location of pain ( $>4 / 5$ regions) and does not exclude an association with another pathology (with lesion or organ damage).

Actually, no treatment can cure FM. Pharmacological management often remains on the prescription of analgesics, antidepressants, and/or antiepileptics, but these therapies are not always effective and often cause undesirable effects $(6,7)$. Nonpharmacological therapies should be advocated as a first-line treatment for patients suffering from FM, such as the practice of a regular physical activity, exercise being "the only 'strong for' therapy-based recommendation in the [European League Against Rheumatism, EULAR] guidelines" (8).

Exercise has clinical relevance over several parameters such as fatigue, physical function, and quality of life (9). However, it can be applied by several ways of training. The American College of Sport Medicine (ACSM) elaborates the recommendations in terms of physical activity, applying the frequency, intensity, time, type, volume, and progression principle (10). For FM, there are no precise recommendations.

Endurance training is recommended by different medical societies for FM management $(8,11-14)$ in the range of two to three sessions a week, allowing to respect a rest period and avoid exacerbation of symptoms, at least for a period of 46 weeks in order to see a decrease in symptoms $(15,16)$ and a change in autonomic function (17). A light-intensity aerobic practice $(15,18)$ does not allow a decrease in symptom intensity, rather indicating a moderate to vigorous intensity physical activity (MVPA) practice. While high-intensity interval training
(HIIT) is increasingly used in several pathologies (19-21), it is only minimally studied in FM. Demonstrating improvements in physical capacities $(22,23)$, HIIT would also be effective for decrease in fatigue $(24,25)$, pain tolerance $(26)$, and opioid system $(27,28)$-three major issues in FM syndrome-without change in pain or disease activity in patients with rheumatic disease (21). Strength training is less documented than aerobic training. Some studies have worked on weight resistance training (two weekly sessions during 16 weeks) with a progressive load from 50 to $80 \%$ of the one-repetition maximum, demonstrating a good compliance and an involvement of force correlated to a decrease of $39 \%$ in pain perception (29). This decrease in pain was also measured in a resistance program followed for 8 weeks with three sessions a week (30). Recently, Andrade et al. (17) identified a correlation between resistance training and reduction in psychological symptoms such as anxiety and depression. Muscle stretching and relaxation exercises also showed an impact on pain (31), pain perception and tolerance (32), quality of life, depression, and anxiety (33) in FM patients.

At equal time of activity, Sanudo et al. (34) noticed additional health benefits of a combined training program compared to a single modality program. A mixed training program is defined as combining at least two types of exercises among aerobics, resistance and flexibility training, and demonstrated an interest in quality of life $(+7 \%)$, fatigue $(-13 \%)$, and physical function $(+11 \%)$, translating the clinical relevance of mixed exercise training for adults with FM (9).

Despite the absence of accurate recommendations on training modalities, as defined by the ACSM, a personalized and mixed training seems to be the best practice according to the EULAR recommendations (8), and it is also this type of training that is recommended for people suffering from chronic pain (35). In France, there are no professional recommendations of good practice for the management of FM. The health insurance recommends to physicians to give advice for the progressive upturn of physical activity for each person with FM, "according to its means."

However, European and French recommendations agree to promote patient education to increase "understanding of the complex nature of the interactions between neurobiological processes, behaviors, and symptoms (36)." It showed beneficial impact on pain and pain catastrophizing (37) despite the different educational strategies. Moreover, an education program would be 


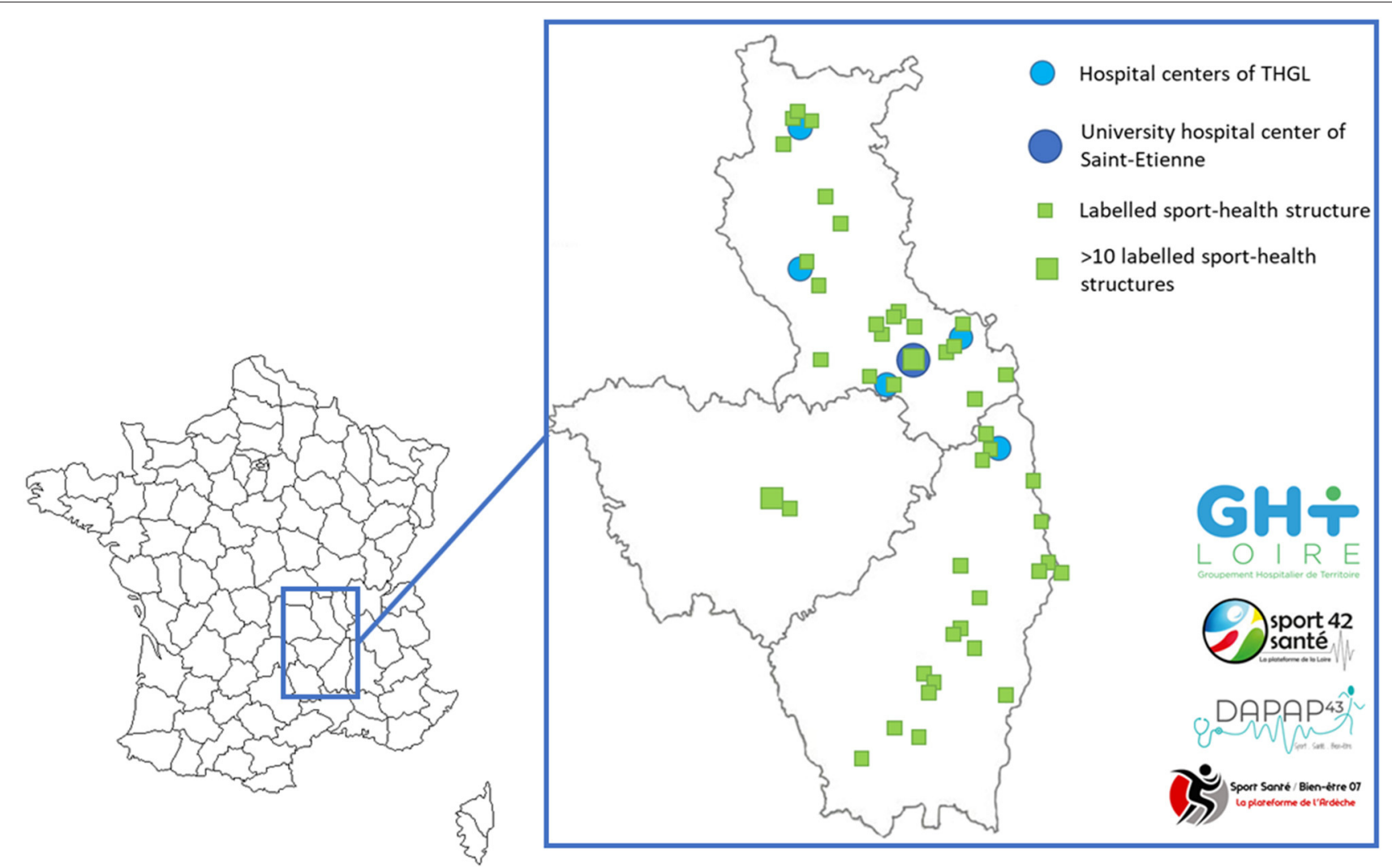

FIGURE 1 | Project collaborators mapping. THGL: Territorial Hospital Group of Loire.

all the more effective if it is combined with an adapted physical activity (APA) program $(38,39)$.

Then, the problem is to know if simple advice for physical activity is sufficient (as recommended by the French health insurance and commonly performed in consultation) or if an adapted and supervised physical activity (as recommend by the EULAR) would not be more beneficial as a first intention. Thus, in this study, we want to optimize the care pathways of patients suffering from FM by offering a supervised APA program added to therapeutic patient education sessions. This is a prospective, multicenter, controlled, randomized, open-label intervention in two groups. The primary objective of this study is to measure the effectiveness of a healthcare organization offering APA for FM patients through an objective measure of physical activity at 1 year (average measured from 7 days of accelerometry). The protocol has been written according to the Standard Protocol Items: Recommendations for Interventional Trials guidelines.

\section{METHODS AND ANALYSIS}

\section{Study Setting}

The FIMOUV study is a collaboration between health professionals of the Territorial Hospital Group of Loire centers in France and physical activity professionals on this territory (Figure 1). Patients will be selected at their follow-up
TABLE 1 | Eligibility criteria.

\begin{tabular}{ll}
\hline Inclusion criteria & Patient over 18 years old \\
& FM syndrome according to ACR criteria (total score WPI \\
& + SSS $\geq 12$ ) \\
& Language skills in oral and written French \\
& Sedentary or low level of activity ( $<150$ min of regular \\
& physical activity per week at the time of inclusion) \\
& Affiliation to a social security scheme \\
& Signing informed consent
\end{tabular}

Exclusion criteria Cardiac or respiratory diseases that contraindicate the practice of physical activity

Severe comorbidities contraindicating the practice of physical activity (rhythm disorders, severe obstructive respiratory failure, gonarthrosis, etc.)

Impossibility of submitting to medical monitoring of the program for geographical, social, or psychological reasons

Patient deprived of liberty or patient under guardianship

FM, fibromyalgia; ACR, American College of Rheumatology; WPI, Widespread Pain Index; SSS, symptom severity scale.

consultation, during which the doctor will give the information leaflet and the consent form. FM patients will then be summoned at the University Hospital of Saint-Etienne (Loire) for the 
inclusion visit (V1). Inclusion and exclusion criteria are detailed in Table 1. We want to compare a supervised training program to physical activity advice, both benefiting from four therapeutic education sessions. Patients are then followed for 1 year (Figure 2).

\section{Interventions}

Training Program

\section{Test Group}

During the first month, the test group benefiting from two weekly exercise sessions of 90 min was supervised by an APA teacher during four consecutive weeks at the hospital. We propose a mixed training program (details on Figure 3), normalized and personalized to each patient profile, as follows:

- Endurance training (Figure 3A): progressive work toward an interval training at an intensity determined during a preliminary cardiorespiratory test (first and second ventilatory threshold, VT1/VT2) and monitored with a heart rate belt connected by Bluetooth (Polar H7, Polar ${ }^{\circledR}$, Kempele, Finland). A continuous training at VT1 can be proposed if the subject's physical capacity does not allow an increase in workloads as they are made on interval training.

- Strength training: whole body circuit training with body weight or small equipment, with at least one exercise soliciting balance, coordination, and/or velocity. Additional loads representing a maximum $50 \%$ of one repetition maximum can be added in case of pain evaluation lower than 4/10 on the Borg modified visual analog scale (VAS) at the start of the session. Modalities of training are detailed in Figure 3B.

- Stretching and relaxation training (Figure 3C): according to the patient's profile, alternation between passive stretching, cardiac coherence for patients with a high levels of anxiety (measured during V1 thanks to the Hospital Anxiety and Depression scale, HADS), and autogenic training initiation if the fatigue evaluation is higher than $7 / 10$ at the start of the session.

From the second to third months, each patient is addressed to a relay structure affiliated to the "Sport-Health Houses" of their department to continue a training program for one to two sessions of 60-90 min weekly. Certified by the Ministry of Sports, these health-sport platforms aim to facilitate the maintenance of physical activities among people experiencing health difficulties, with an individualized follow-up. Additionally, a training followup booklet will be delivered to ensure the continuity and attendance of training sessions (supervised and autonomous) and evaluate their efficacy on pain and fatigue symptoms thanks to the VAS.

\section{Control Group}

The control group receives advice and recommendations for independent physical activity practice at home in order to reach $30 \mathrm{~min}$ of moderate intensity physical activity, 5 days a week, as recommended by the World Health Organization (WHO) (current clinical practice).

\section{Therapeutic Education Program}

Therapeutic patient education programs aim to acquire selfcare skills and mobilize or acquire psychosocial skills. In this protocol, we propose four sessions of $2 \mathrm{~h}$, in groups of three to eight patients. The two groups benefit from (1) one session about physiopathology and symptoms of FM by the internal medicine service, (2) one session on physical inactivity and sedentary lifestyle by the myology unit team, (3) one session on the resumption of professional activity by the occupational health service, and (4) one session about the psychological impact and daily routine by the pain center (Figure 3D). All sessions are driven by the coordinating nurse who makes the link between patients and the other health professionals. An individual therapeutic education session will be proposed at $\mathrm{V} 1$ and V3 via motivational interviewing led by one of the investigator doctors and the nurse/APA teacher duo in order to optimize the durability of the practice of regular physical activity.

\section{Assessment Measures}

The primary outcome of interest will be the long-term therapeutic adherence of FM patients evaluated from an objective measure of physical activity (mean measured from 7 days of accelerometry; Actigraph GT3x, Pensacola, Florida, USA) 1 year after the setup of the organization of care offering initiation and support of APA during 3 months. This sustainability will result in a level of physical activity $\geq 7.5 \mathrm{MET}-\mathrm{h} /$ week at 1 year, corresponding to $150 \mathrm{~min}$ of moderate intensity physical activity per week, which is consistent with WHO recommendations.

The secondary objectives will evaluate the impact on the lifestyle (average time of physical activity and sedentary behavior, measured by accelerometry during 7 days), health status, fatigue, sleep, psychic distress, muscular strength and endurance, physical capacity, tolerance to pain and fatigue during training sessions, membership to physical activity, evaluation of the differential cost-effectiveness ratio, and finally an estimate of the budgetary impact of this care strategy.

Details and timetable are shown in Table 2.

\section{Sample Size}

Population size is calculated on an expected difference of $20 \%$ of the level of MVPA ( $\geq 3$ METs for metabolic equivalent tasks). Estimating that at baseline, patients are at 3.9 MET-h/week with a standard deviation between 2 and 4 (40), for an alpha risk of $5 \%$ and a potency set at $90 \%$, we need to include 150 patients per group; adding an expected dropout rate of $10 \%$, it is necessary to include 165 patients per group to meet the main goal, which means a total of 330 patients.

\section{Randomization and Data Collection}

Randomization and data collection will be centralized via the EnnoV Clinical platform (electronical randomization, Paris, France) and filled in by the referral doctor and the nurse/APA teacher duo. Only investigators and technicians of the study have access to these data.

Patients will be randomized after a multidisciplinary consulting meeting carried out in the presence of the investigators of the study, the referring doctor and, if necessary, 

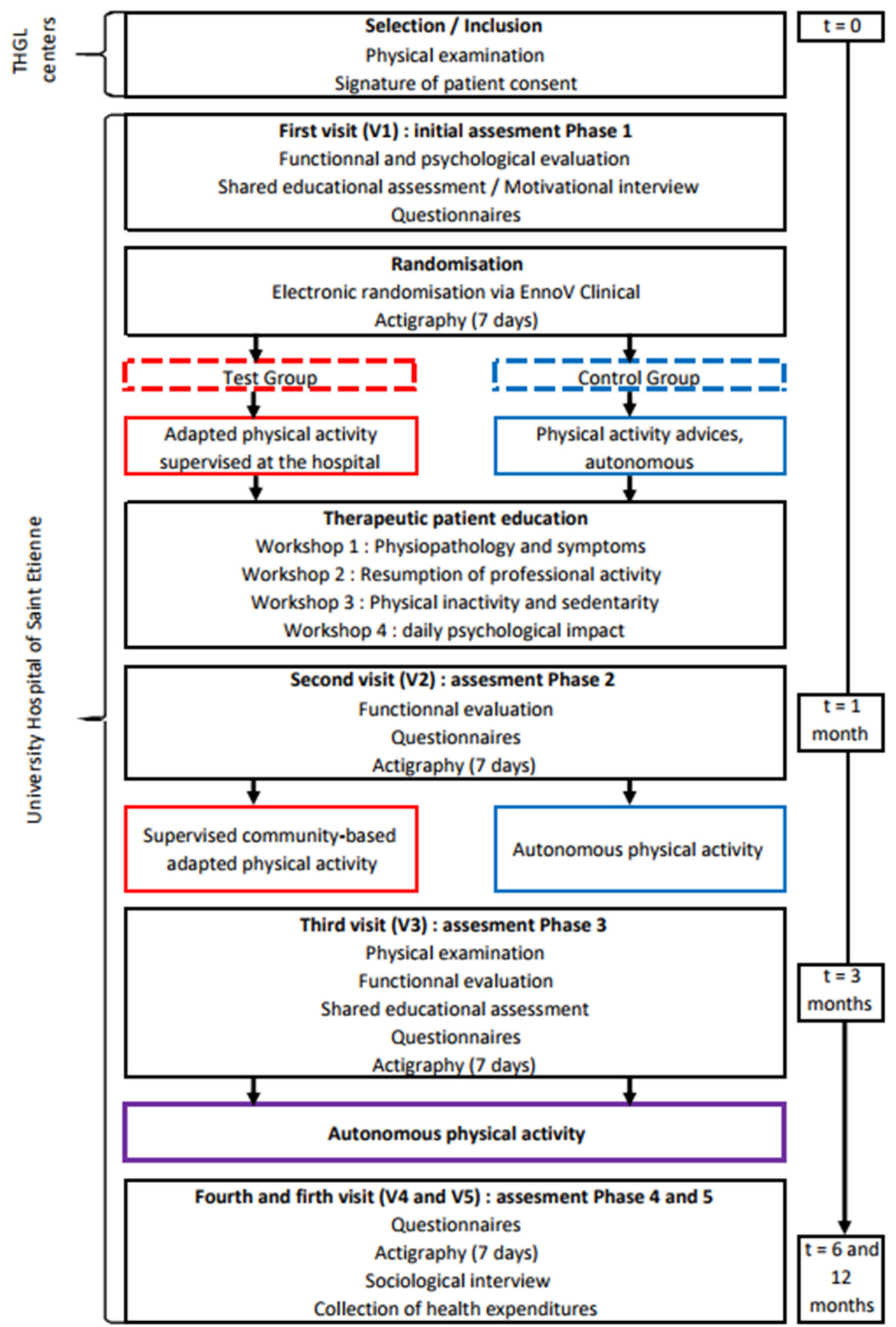

FIGURE 2 | Study design of FIMOUV. THGL, Territorial Hospital Group of Loire. 


\section{A}

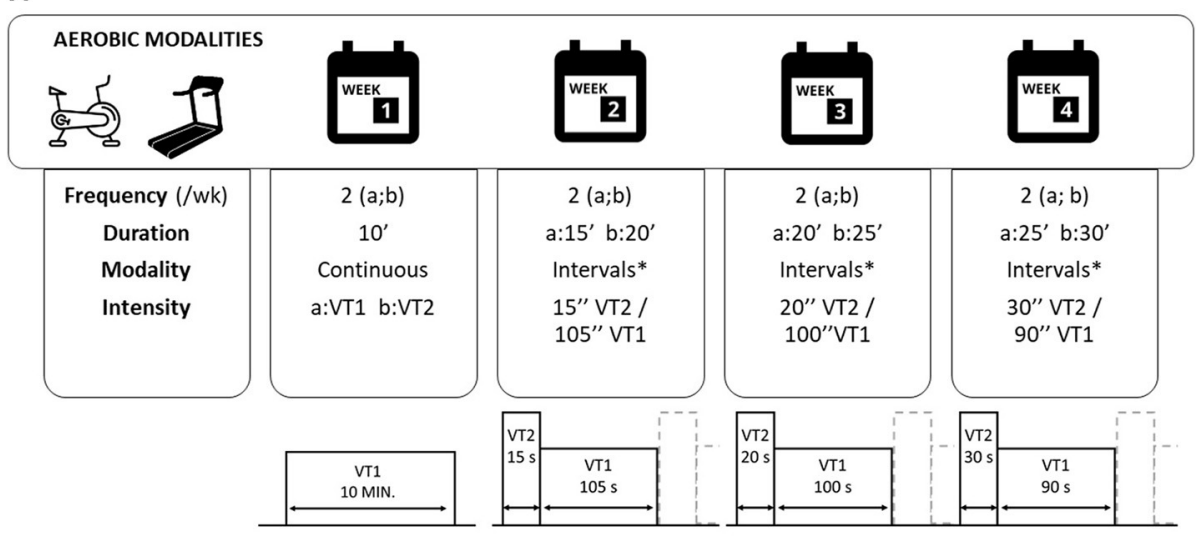

B

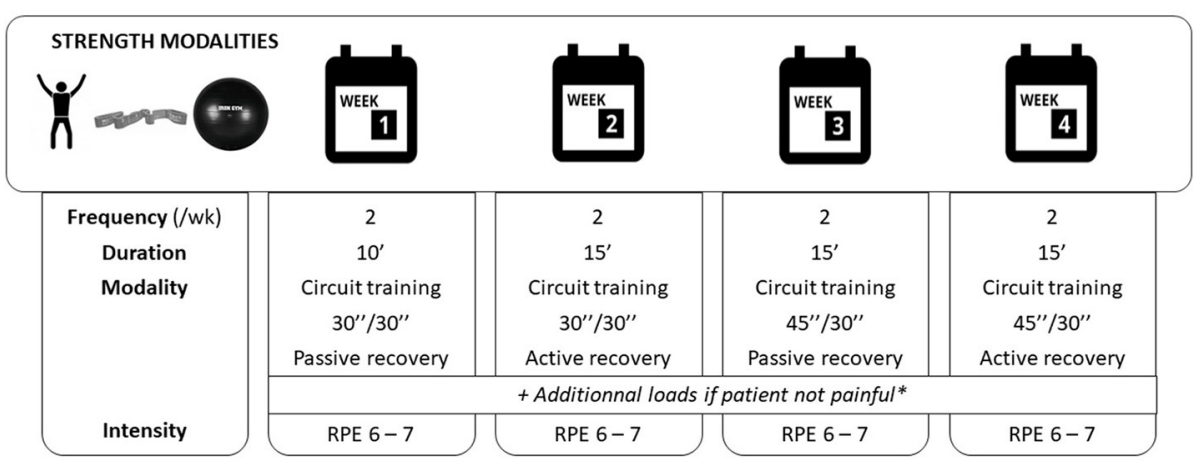

C

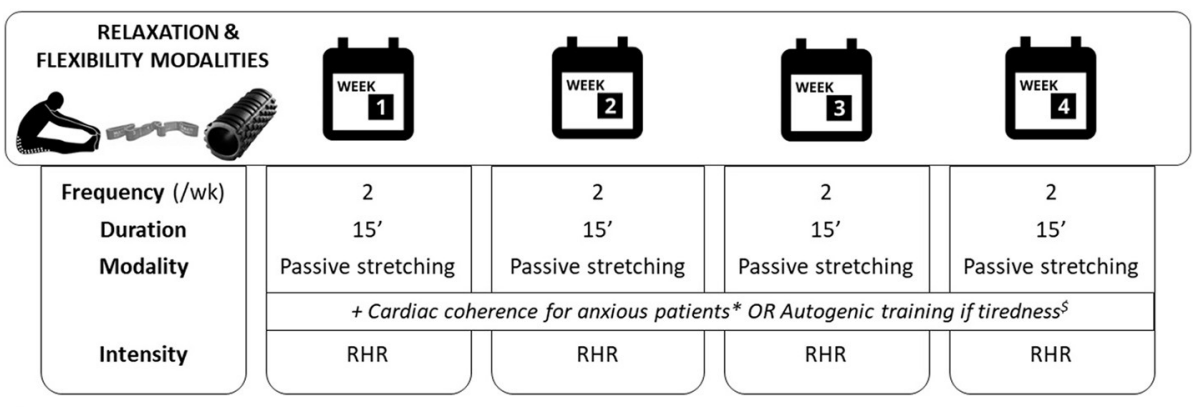

D

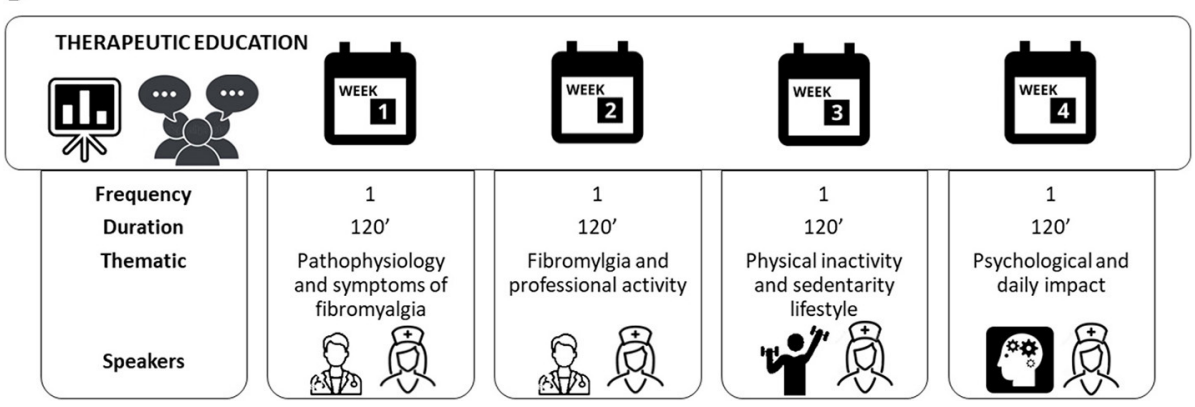

FIGURE 3 | FIMOUV program intervention. (A) Modalities of aerobic training during the first month of intervention. Each session starts with 10 min warmup and ends with 10 min cooldown. *A continuous training at VT1 can be proposed if the subject's physical capacity does not allow the increase in workloads, as they are made on interval training. (a;b) corresponds to each session of the week; VT1: ventilatory threshold 1; VT2: ventilatory threshold 2. (B) Modalities of strength training during the first month of intervention. *Load maximum $50 \%$ of 1 -repetition maximum if pain evaluation $<4 / 10$ at the start of the session. RPE: rating of perceived exertion, Borg modified scale. (C) Modalities of relaxation and flexibility training during the first month of intervention. ${ }^{*}$ Hospital Anxiety and Depression Scale $>8$ for anxiety item.

$\$$ Fatigue evaluation $>7 / 10$ at the start of the session. RHR: resting heart rate. (D) Modalities of therapeutic education program during the first month of intervention. 
TABLE 2 | Assessment measures timetable.

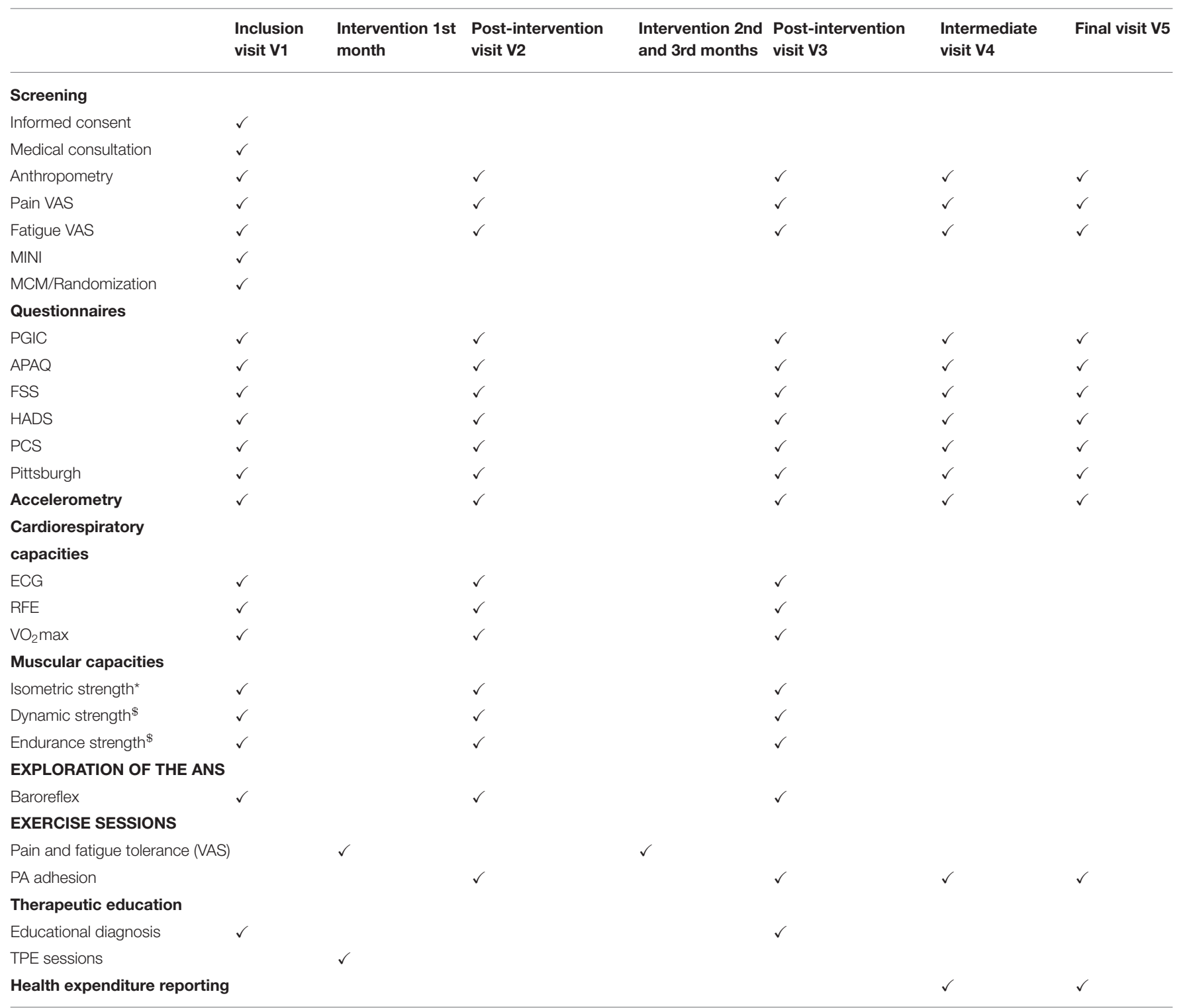

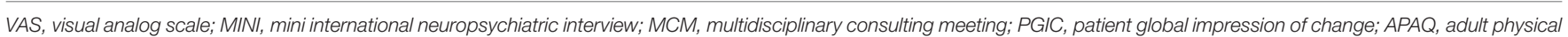

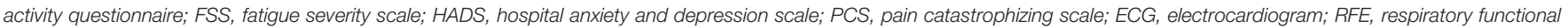
exploration; $\mathrm{VO}_{2} \max$, maximum oxygen consumption; ANS, autonomic nervous system; PA, physical activity; TPE, therapeutic patient education. ${ }^{*}$ arms and legs. ${ }^{\$}$ legs.

with their general practitioner, before the program starts. This meeting aims to decide about the optimal care for the patient. It will be decided if the patient can follow an exercise training. In case of contraindication, the patient will not be randomized into the study.

\section{Statistical Analysis}

The included population will first be described globally and in groups (test or control), the comparability of the groups will be verified. For quantitative variables, Student's $T$-tests or rank tests (in case of non-normal distribution) will be implemented. The normality of the variables will be checked beforehand with a Shapiro-Wilk test. For qualitative variables, chi-square or Fisherexact tests (if theoretical numbers are insufficient) will be used.
The results will be considered significant at the $5 \%$ threshold. The analysis will be carried out in intent-to-treat. An analysis of the main endpoint will be done per protocol. Patients who do not perform $85 \%$ of the exercises will be excluded from per-protocol statistical analysis. In addition, we will lead a qualitative study through semistructured individual interviews conducted by a sport and health sociologist. Thirty patients will be recruited in each of the two groups $(41,42)$. A full transcript will be followed by a thematic and comparative analysis of the cross-data content.

\section{DISCUSSION}

This study aims to evaluate the efficacy of an optimized care organization within the Loire Territory Hospital Group, 
illustrated by the sustainability of therapeutic adherence of FM patients. This interventional research will allow us to assess the evolution of behaviors in physical activity after a FM syndrome management based solely on patient education or based on a supervised and adapted practice of physical activity associated with this same therapeutic education program.

Supervised exercise allows a better participation and adhesion to physical activity in sedentary women (43) and an improvement in psychological well-being determined by the result of HADS in FM (44). Improvements up to two times greater than those of unsupervised training can be observed, however without necessarily being maintained for the long-term at 1 year (45). A recent study has explored the experience of the transition from a supervised exercise in the hospital to a community-based unsupervised program in prostate cancer patients, emphasizing the importance of a structured, accompanied and over time monitored relay (46). Dnes et al. (47) identified the barriers and facilitators to participating in community-based exercise opportunities from the perspective of adults with chronic pain, including FM. The main factors identified were participation in groups with similar levels of capabilities, delivered by an instructor able to tailor exercises to chronic pain and recommended by their healthcare provider. Also, in this project, the implementation of physical activity in community programs, chosen by the patient himself/herself according to his/her preferences and availability, could strengthen the integration of physical activity into daily life through the connection with the sports health centers, which have the role of labeling the healthsports club, with groups specific to chronic pathologies delivered by trained instructors.

The combination of education and physical activity demonstrated its efficacy on management of several FM symptoms. However, the level of physical activity practice in the short-term after the intervention and at the long-term at 1 year has been barely studied. Physical activity monitors are interesting tools to better understand the role of physical activity in rheumatic disease populations (48). For example, the use of a pedometer to measure the activity in FM patients after a multidisciplinary intervention based on cognitive behavioral therapy and physical therapy demonstrated an increase in exercise capacity and regularity (49). Others studies evaluated the physical activity behavior in FM patients compared to healthy subjects, and the link between their behavior and their symptoms $(40,50)$. However, to our knowledge, no study has evaluated the impact of its intervention on objective data for measuring physical activity and sedentary behavior via accelerometry among FM patients.

Qualitative analysis of the project will provide data for evaluation of the acceptability and the feasibility of our intervention, according to the patients' experiences. This analysis will allow us to better understand the barriers and facilitators that can be used to design an intervention more suited to the expressed needs of the participants.
To conclude, the multidisciplinary management proposed must contribute to the construction of a solid therapeutic alliance between the patient and the management of its FM syndrome and thus give the initial medical prescription of APA the appearance of a therapeutic project. It is also a question of strengthening the city-hospital link by (1) developing an optimal care sector, (2) which combines therapies validated by scientific societies, (3) including APA that we wish to place at the center of a multidisciplinary and consensual care organization, and (4) which relies on existing and efficient structures for many chronic pathologies, with (5) an objective of sustainability within the largest national territory hospital group.

\section{ETHICS STATEMENT}

The protocol is in accordance with ethical principles established by the 18th World Medical Assembly (Helsinki 1964) and was approved by the institutional review board (CPP SudMéditerranée II, France). Each patient will be awarded an information notice explaining the study and must sign an informed consent form before being able to participate in the research.

\section{AUTHOR CONTRIBUTIONS}

The FIMOUV investigators from University Hospital of SaintEtienne contributed to the conception and design of the study. CCo, M-PV, MA, and DH contributed to the acquisition of data. CCo wrote the first draft of the manuscript. $\mathrm{DH}$ provided critical revision for intellectual content and oversight. All the authors reviewed and approved the final version of the manuscript.

\section{FUNDING}

This study was funded after the calls for projects of the University Hospital of Saint-Etienne (AOL 2018) and the research program on the performance of the healthcare system (PREPS 2018) proposed by the Ministry of Solidarity and Health (France).

\section{ACKNOWLEDGMENTS}

We thank all of the FIMOUV investigators: Elodie Breda, Orane Barbet, Jonathan Bazard, Paul Calmels, Jean-Philippe Camdessanche, Pascal Cathebras, Clémence Charroin, JeanPaul Chaussinand, Christelle Creac'h, Isabelle Fayolle-Minon, Léonard Feasson, Luc Fontana, Julie Goutte, Malorie Grange, David Hupin, Martin Killian, Béatrice Lietar, Frédéric Lucht, Hubert Marotte, Catherine Massoubre, Paul Mirkovic, Colas Morel-Prieur, Malou Navez, Philippe Nayme, Mariana Pernea, Aurélie Robles, Frédéric Roche, Bachir Sahi, Charles Schweckler, Thierry Thomas, and Delphine Vergnon Miszczycha. 


\section{REFERENCES}

1. Vincent A, Lahr BD, Wolfe F, Clauw DJ, Whipple MO, Oh TH, et al. Prevalence of fibromyalgia: a population-based study in olmsted county, minnesota, utilizing the rochester epidemiology project. Arthr Care Res (Hoboken). (2013) 65:786-92. doi: 10.1002/acr.21896

2. Wolfe F, Smythe HA, Yunus MB, Bennett RM, Bombardier C, Goldenberg DL, et al. The american college of rheumatology 1990 criteria for the classification of fibromyalgia. Arthr Rheum. (1990) 33:160-72. doi: 10.1002/art.1780330203

3. Abeles AM, Pillinger MH, Solitar BM, Abeles M. Narrative review: the pathophysiology of fibromyalgia. Ann Intern Med. (2007) 146:72634. doi: 10.7326/0003-4819-146-10-200705150-00006

4. Wolfe F, Clauw DJ, Fitzcharles M-A, Goldenberg DL, Häuser W, Katz RL, et al. Revisions to the 2010/2011 fibromyalgia diagnostic criteria. Sem Arthr Rheum. (2016) 46:319-29. doi: 10.1016/j.semarthrit.2016.08.012

5. Wolfe F, Clauw DJ, Fitzcharles M-A, Goldenberg DL, Katz RS, Mease P, et al. The American college of rheumatology preliminary diagnostic criteria for fibromyalgia and measurement of symptom severity. Arthr Care Res. (2010) 62:600-10. doi: 10.1002/acr.20140

6. Choy E, Marshall D, Gabriel ZL, Mitchell SA, Gylee E, Dakin HA. A systematic review and mixed treatment comparison of the efficacy of pharmacological treatments for fibromyalgia. Semin Arthr Rheum. (2011) 41:335-345.e6. doi: 10.1016/j.semarthrit.2011.06.003

7. Roskell NS, Beard SM, Zhao Y, Le TK. A meta-analysis of pain response in the treatment of fibromyalgia. Pain Practice. (2011) 11:51627. doi: 10.1111/j.1533-2500.2010.00441.x

8. Macfarlane GJ, Kronisch C, Dean LE, Atzeni F, Häuser W, Fluß E, et al. EULAR revised recommendations for the management of fibromyalgia. Ann Rheum Dis. (2017) 76:318-28. doi: 10.1136/annrheumdis-2016-209724

9. Bidonde J, Busch AJ, Schachter CL, Webber SC, Musselman KE, Overend TJ, et al. Mixed exercise training for adults with fibromyalgia. Cochrane Database Syst Rev. (2019) 5:CD013340. doi: 10.1002/14651858.CD013340

10. American College of Sports Medicine. ACSM's Guidelines for Exercise Testing and Prescription. 9th ed. Philadelphia, PA: Lippincott Williams \& Wilkins (2014).

11. Fitzcharles M-A, Ste-Marie PA, Goldenberg DL, Pereira JX, Abbey S, Choinière $\mathrm{M}$, et al. Canadian Guidelines for the diagnosis and management of fibromyalgia syndrome: executive summary. Pain Res Manag. (2013) 18:11926. doi: $10.1155 / 2013 / 918216$

12. Buckhardt C, Goldenberg D, Crofford L, Gerwin R, Gowens S, Jackson K, et al. Guideline for the Management of Fibromyalgia Syndrome Pain in Adults and Children. Glenview, IL : American Pain Society. (2005). p. 109. (Clinical practice guideline (American Pain Society), no. 4.).

13. Häuser W, Arnold B, Eich W, Felde E, Flügge C, Henningsen P, et al. Management of fibromyalgia syndrome - an interdisciplinary evidence-based guideline. Ger Med Sci. (2008) 6:383. doi: 10.3238/arztebl.2009.0383

14. Ablin JN, Amital H, Ehrenfeld M, Aloush V, Elkayam O, Langevitz P, et al. Guidelines for the diagnosis and treatment of the fibromyalgia syndrome. Harefuah. (2013) 152:742-7:751, 750.

15. Häuser W, Klose P, Langhorst J, Moradi B, Steinbach M, Schiltenwolf M, et al. Efficacy of different types of aerobic exercise in fibromyalgia syndrome: a systematic review and meta-analysis of randomised controlled trials. Arthritis Res Ther. (2010) 12:R79. doi: 10.1186/ar3002

16. Busch AJ, Barber KAR, Overend TJ, Peloso PMJ, Schachter CL. Exercise for treating fibromyalgia syndrome. Cochrane Database of Systematic Reviews. (2007) (4). doi: 10.1002/14651858.CD003786.pub2

17. Andrade A, Vilarino GT, Serafim TT, Pereira Júnior AA, de Souza CA, Sieczkowska SM. Modulation of Autonomic Function by Physical Exercise in Patients with Fibromyalgia Syndrome: A Systematic Review. PM R. (2019) 11:1121-31. doi: 10.1002/pmrj.12158

18. Black CD, O'Connor PJ, McCully KK. Increased daily physical activity and fatigue symptoms in chronic fatigue syndrome. Dyn Med. (2005) 4:3. doi: 10.1186/1476-5918-4-3

19. Mijwel S, Backman M, Bolam KA, Jervaeus A, Sundberg CJ, Margolin S, et al. Adding high-intensity interval training to conventional training modalities: optimizing health-related outcomes during chemotherapy for breast cancer: the OptiTrain randomized controlled trial. Breast Cancer Res Treat. (2018) 168:79-93. doi: 10.1007/s10549-017-4571-3
20. Pavy B, Iliou MC, Vergès-Patois B, Brion R, Monpère C, et al. Exercise, Sports and Rehabilitation Team. French Society of Cardiology guidelines for cardiac rehabilitation in adults. Arch Cardiovasc Dis. (2012) 105:30928. doi: 10.1016/j.acvd.2012.01.010

21. Sandstad J, Stensvold D, Hoff M, Nes BM, Arbo I, Bye A. The effects of high intensity interval training in women with rheumatic disease: a pilot study. Eur J Appl Physiol. (2015) 115:2081-9. doi: 10.1007/s00421-015-3186-9

22. Santen M van, Bolwijn P, Landewé R, Verstappen F, Bakker C, Hidding A, et al. High or low intensity aerobic fitness training in fibromyalgia: does it matter? J Rheum. (2002) 29:582-7.

23. Mannerkorpi K, Nordeman L, Cider $\AA$, Jonsson G. Does moderate-tohigh intensity Nordic walking improve functional capacity and pain in fibromyalgia? A prospective randomized controlled trial. Arthritis Res Ther. (2010) 12:R189. doi: 10.1186/ar3159

24. Sveaas SH, Berg IJ, Fongen C, Provan SA, Dagfinrud H. High-intensity cardiorespiratory and strength exercises reduced emotional distress and fatigue in patients with axial spondyloarthritis: a randomized controlled pilot study. Scand J Rheum. (2018) 47:117-21. doi: 10.1080/03009742.2017.1347276

25. Thomsen RS, Nilsen TIL, Haugeberg G, Bye A, Kavanaugh A, Hoff M. Impact of high-intensity interval training on disease activity and disease in patients with psoriatic arthritis: a randomized controlled trial. Arthritis Care Res. (2019) 71:530-7. doi: 10.1002/acr.23614

26. O'Leary TJ, Collett J, Howells K, Morris MG. High but not moderate-intensity endurance training increases pain tolerance: a randomised trial. Eur J Appl Physiol. (2017) 117:2201-10. doi: 10.1007/s00421-017-3708-8

27. Saanijoki T, Tuominen L, Tuulari JJ, Nummenmaa L, Arponen E, Kalliokoski $\mathrm{K}$, et al. Opioid release after high-intensity interval training in healthy human subjects. Neuropsychopharmacology. (2018) 43:24654. doi: 10.1038/npp.2017.148

28. Zubieta JK, Smith YR, Bueller JA, Xu Y, Kilbourn MR, Jewett DM, et al. Regional mu opioid receptor regulation of sensory and affective dimensions of pain. Science. (2001) 293:311-5. doi: 10.1126/science.1060952

29. Figueroa A, Kingsley JD, McMillan V, Panton LB. Resistance exercise training improves heart rate variability in women with fibromyalgia. Clin Phys Funct Imaging. (2008) 28:49-54. doi: 10.1111/j.1475-097X.2007.00776.x

30. Bircan Ç, Karasel SA, Akgün B, El Ö, Alper S. Effects of muscle strengthening versus aerobic exercise program in fibromyalgia. Rheumatol Int. (2008) 28:527-32. doi: 10.1007/s00296-007-0484-5

31. Matsutani LA, Marques AP, Ferreira E a. G, Assumpção A, Lage LV, Casarotto RA, et al. Effectiveness of muscle stretching exercises with and without laser therapy at tender points for patients with fibromyalgia. Clin Exp Rheumatol. (2007) 25:410-5.

32. Assumpção A, Matsutani LA, Yuan SL, Santo AS, Sauer J, Mango P, et al. Muscle stretching exercises and resistance training in fibromyalgia: which is better? A three-arm randomized controlled trial. Eur J Phys Rehabil Med. (2018) 54:663-70. doi: 10.23736/S1973-9087.17.04876-6

33. Gavi MBRO, Vassalo DV, Amaral FT, Macedo DCF, Gava PL, Dantas EM, et al. Strengthening exercises improve symptoms and quality of life but do not change autonomic modulation in fibromyalgia: a randomized clinical trial. PLoS ONE. (2014) 9:907676. doi: 10.1371/journal.pone.0090767

34. Sañudo B, Galiano D, Carrasco L, Blagojevic M, Hoyo M de, Saxton J. Aerobic Exercise Versus Combined Exercise Therapy in Women With Fibromyalgia Syndrome: A Randomized Controlled Trial. Archives of Physical Medicine and Rehabilitation. (2010) 91:1838-43. doi: 10.1016/j.apmr.2010.09.006

35. O'Riordan C, Clifford A, Van De Ven P, Nelson J. Chronic neck pain and exercise interventions: frequency, intensity, time, and type principle. Arch Phys Med Rehab. (2014) 95:770-83. doi: 10.1016/j.apmr.2013.11.015

36. Hassett AL, Gevirtz RN. Nonpharmacologic treatment for fibromyalgia: patient education, cognitive-behavioral therapy, relaxation techniques, and complementary and alternative medicine. Rheum Dis Clin North Am. (2009) 35:393-407. doi: 10.1016/j.rdc.2009.05.003

37. García-Ríos MC, Navarro-Ledesma S, Tapia-Haro RM, ToledanoMoreno S, Casas-Barragán A, Correa-Rodríguez M, et al. Effectiveness of health education in patients with fibromyalgia: a systematic review. Eur J Phys Rehabil Med. (2019) 55:301-13. doi: 10.23736/S1973-9087.19. 05524-2

38. Rooks DS, Gautam S, Romeling M, Cross ML, Stratigakis D, Evans B, et al. Group exercise, education, and combination self-management in women 
with fibromyalgia: a randomized trial. Arch Intern Med. (2007) 167:2192200. doi: 10.1001/archinte.167.20.2192

39. King SJ, Wessel J, Bhambhani Y, Sholter D, Maksymowych W. The effects of exercise and education, individually or combined, in women with fibromyalgia. J Rheum. (2002) 29:2620-7.

40. McLoughlin MJ, Colbert LH, Stegner AJ, Cook DB. Are women with fibromyalgia less physically active than healthy women? Med Sci Sports Exerc. (2011) 43:905-12. doi: 10.1249/MSS.0b013e3181fcalea

41. Mason M. Sample size and saturation in phd studies using qualitative interviews. Forum: Qualit Soc Re. (2010). 11:8. Available online at: http://nbnresolving.de/urn:nbn:de:0114-fqs100387

42. Griffin A, Hauser JR. The voice of the customer. Market Sci. (1993) 12:127. doi: $10.1287 / \mathrm{mksc} \cdot 12.1 .1$

43. Cox KL, Burke V, Gorely TJ, Beilin LJ, Puddey IB. Controlled comparison of retention and adherence in home- vs center-initiated exercise interventions in women ages 40-65 years: the S.W.E.A.T. Study (Sedentary Women Exercise Adherence Trial). Prevent Med. (2003) 36:17-29. doi: 10.1006/pmed.2002.1134

44. Ramsay C, Moreland J, Ho M, Joyce S, Walker S, Pullar T. An observer-blinded comparison of supervised and unsupervised aerobic exercise regimens in fibromyalgia. Rheumatology (Oxford). (2000) 39:5015. doi: 10.1093/rheumatology/39.5.501

45. Deyle GD, Allison SC, Matekel RL, Ryder MG, Stang JM, Gohdes DD, et al. Physical therapy treatment effectiveness for osteoarthritis of the knee: a randomized comparison of supervised clinical exercise and manual therapy procedures versus a home exercise program. Phys Ther. (2005) 85:130117. doi: $10.1093 / \mathrm{ptj} / 85.12 .1301$

46. Schmidt MLK, Østergren P, Cormie P, Ragle A-M, Sønksen J, Midtgaard J. "Kicked out into the real world": prostate cancer patients' experiences with transitioning from hospital-based supervised exercise to unsupervised exercise in the community. Support Care Cancer. (2019). 27:199-208. doi: 10.1007/s00520-018-4306-y
47. Dnes N, Coley B, Frisby K, Keller A, Suyom J, Tsui C, et al. "A little bit of a guidance and a little bit of group support": a qualitative study of preferences, barriers, and facilitators to participating in community-based exercise opportunities among adults living with chronic pain. Disabil Rehab. (2020) 1-10. doi: 10.1080/09638288.2020. 1742801

48. Pellegrini CA, Powell SM, Mook N, DeVivo K, Ehrlich-Jones L. Use of physical activity monitors in rheumatic populations. Curr Rheumatol Rep. (2018) 20:73. doi: 10.1007/s11926-018-0786-5

49. Salvat I, Zaldivar P, Monterde S, Montull S, Miralles I, Castel A. Functional status, physical activity level, and exercise regularity in patients with fibromyalgia after Multidisciplinary treatment: retrospective analysis of a randomized controlled trial. Rheumatol Int. (2017) 37:37787. doi: 10.1007/s00296-016-3597-x

50. Kop WJ, Lyden A, Berlin AA, Ambrose K, Olsen C, Gracely RH, et al. Ambulatory monitoring of physical activity and symptoms in fibromyalgia and chronic fatigue syndrome. Arthr Rheum. (2005) 52:296-303. doi: 10.1002/art. 20779

Conflict of Interest: The authors declare that the research was conducted in the absence of any commercial or financial relationships that could be construed as a potential conflict of interest.

Copyright (C) 2021 Colas, Goutte, Creac'h, Fontana, Vericel, Manzanares, Peuriere, Akrour, Martin, Presles, Barth, Guyot, Garros, Trombert, Massoubre, Roche, Féasson, Marotte, Cathebras and Hupin. This is an open-access article distributed under the terms of the Creative Commons Attribution License (CC BY). The use, distribution or reproduction in other forums is permitted, provided the original author(s) and the copyright owner(s) are credited and that the original publication in this journal is cited, in accordance with accepted academic practice. No use, distribution or reproduction is permitted which does not comply with these terms. 